\title{
Polarization of Light and some Technical Applications
}

\author{
By Prof. A. F. C. Pollard
}

$\mathrm{W}$ HATEVER may be the nature of light, it is convenient to regard it as the propagation of an electro-magnetic disturbance in the ether. In an isotropic medium, free of electric charge, the electric and magnetic vectors are perpendicular to one another and to the direction of propagation. Either of these may be selected as the periodic vector-the light vector-associated with the disturbance, but it is usual to define the plane of polarization as that containing the magnetic force with the electric force perpendicular to it.

There are three fundamental qualities which are of major importance to the control of light.

The wave-length, which determines the frequency or colour and the amplitude upon which the intensity of light depends, are two of these qualities which have been under almost unlimited control for centuries. But the third-the direction and type of the transverse vibration-has continued to be under very limited control up to the present time only since 1669 when Erasmus Bartholinus observed the double-refraction of Iceland spar. A few years after the publication of Bartholinus's work, Huyghens indicated the distinctive character of the two beams transmitted by the spar, and Newton's discussion on the two-sidedness of the beams led to their subsequent description as polarized light. Curiously enough, this great discovery lay dormant as an isolated fact in science until about 1808 , when Malus accidentally observed that the light reflected from the glass windows of the Luxembourg palace in Paris was polarized. Biot, making use of Malus's discovery, constructed his reflecting polariscope, which was one of the first devices to produce polarized light somewhat inefficiently by reflection.

It was not until 1828, however, that the optical anisotropy of Iceland spar was utilized by William Nicol as an efficient means for producing a plane polarized beam of light. With his famous prism, one of the beams-the ordinary ray-is shunted to one side, whilst the other-the extraordinary ray-is transmitted with but slight absorption as a pencil of wholly polarized light.

The working aperture of Nicol's prism, however, is limited by the size of optically perfect crystals of the spar, and the largest which has been made has an aperture of about 4 inches. Usually, nicols are much smaller than this, and even moderately sized optically perfect crystals of the spar have already become scarce.

Commencing with Foucault in 1857 a number of investigators-Dove, Hartnack and Prazmouski, Jamin, Glan, Feussner, Zenker, Abbe, Ahrens, Silvanus Thompson and others-have devised various prisms with the object of taking every advantage to increase the aperture and economize the precious spar.

Nothing has yet been devised which equals the nicol for transparency and for the perfection of the polarization of the transmitted light; but when large apertures are required the only alternative has been the reflecting polarizer, which produces an imperfectly polarized beam with much loss of light. The recent work of E. H. Land, who makes use of the dichroic properties of certain substances, has, however, resulted in simple means for filtering out of common light a component with high percentage plane polarization by transmission through any sized aperture desired.

To understand Land's invention fully, it must be remembered that optical anisotropy may be manifest as double refraction in which the ordinary and extraordinary rays are transmitted with unequal absorption, when the difference of the coefficients of absorption may be called true dichroism.

The decrease in the intensity of the light when passing through an optically anisotropic plate may be partly due to scatter and partly due to true absorption. The difference of the coefficients of scattering is called 'dityndallism', and this with the true dichroism makes up the total dichroism. In most crystalline substances the dityndallism is negligible, but in colloidal anisotropic systems it may be important.

The absorption depends not only upon the thickness of the plate but also upon the wave. length, and consequently in nearly all dichroic crystals the transmitted light is coloured. The outstanding and historical example of a dichroic uniaxial crystal, in which the transmitted light is strongly polarized and nearly colourless, is tourmaline. In certain tourmalines the ordinary ray is completely absorbed, but the extraordinary ray vibrating in a plane parallel to the trigonal axis is transmitted unfortunately with so much absorption as to render most good polarizing tourmalines useless in feeble illumination. 
In 1851 Dr. William Bird Herapath ${ }^{1}$ discovered a remarkable compound of iodine and quinine sulphate, iodosulphate of quinine, $4 \mathrm{Qu} .3 \mathrm{H}_{2} \mathrm{SO}_{4}$. $2 \mathrm{HI} . \mathrm{I}_{4} \cdot 6 \mathrm{H}_{2} \mathrm{O}$, which aroused great interest at the time and was afterwards called Herapathite by Haidinger. Later, he discovered a similar dichroic compound of strychnine.

The double refracting Herapathite can be crystallized in minute hexagonal plates, and it has been stated that such a plate $1 / 200$ inch thick will completely absorb one of the rays, permitting the other to be transmitted with scarcely perceptible absorption. Large crystals cannot be made, though some were made large enough by Dr. Herapath to be used by Beale as polarizing components in the microscope ${ }^{2}$. These crystals were used for the microscope very shortly after their discovery, but their permanence could not be depended upons. If exposed to the air or mounted in Canada balsam dissolved in xylol, the iodine disappears and the crystals lose their polarizing properties. They can be preserved, however, in Canada balsam dissolved in ether.

I have succeeded in making crystals $2-3 \mathrm{~mm}$. in diameter, but they were not flat and far too friable to be ground into parallel plates.

Dr. Herapath's arresting discovery seems to have passed into oblivion until Land succeeded in making a thin sheet of nitrocellulose packed with ultra-microscopic crystals of Herapathite with their optic axes all parallel to one another. Such a sheet behaves like a single extended crystal, with the difference that since the crystalline particles overlap in the direction of the thickness of the film the transmitted beam is absorbed more than it would be in a single crystal and the sheets have a smoky brown appearance.

In his patent specification ${ }^{4}$, Land describes processes by which these sheets may be made. One way is to prepare a gel-like mass containing Herapathite which is thoroughly mixed with a viscous nitrocellulose or cellulose acetate solution. Since the particles are asymmetrical, they will orient themselves in the same direction when the viscous mass is extruded through a slit-like die. But the retarded flow of the outer surfaces of the material during its passage through the die as compared with the flow of deeper layers will give rise to surface shear upsetting the regular arrangement of the crystals. To overcome this difficulty a layer of the charged plastic is placed between two layers of uncharged plastic before extrusion through the die. The middle charged layer then flows with practically uniform velocity over the cross section and the particles are uniformly distributed parallel to one another.

Another method is to place the viscous polarizing material in contact with a substratum of viscous non-polarizing material, such as fluid celluloid or other colourless oily ester, spread upon a glass plate or sheet of celluloid in such a way as to stretch the polarizing medium. Orientation of the particles may also be brought about by subjecting a diluted colloidal suspension to an electric or magnetic field during the setting of the mass.

It is by some such means described by Land that the Polaroid Corporation of Boston, U.S.A., has succeeded in manufacturing the 'Polaroid' sheets of polarizing material now on the market. Other firms have secured the right to employ the material in special applications. Thus, one firm supplies Polaroid analysers and polarizers for the microscope, as well as for projecting lanterns and other instruments. Another manufactures ophthalmic instruments employing Polaroid. The Eastman Kodak Co. employs the material for photographic purposes under the trade name of 'Pola Screen'; the Royal Photographic Society has awarded the Hood Medal to Mr. Edwin H. Land for his meritorious invention.

There are two grades of Pola screen at present available-Types I and II. Type I consists of sheet material cemented between glass plates of $A$ or $B$ quality intended for use over the camera lens. The $A$ quality glass plates are optical flats hand surfaced of the highest quality, and the $B$ plates are of optical glass of good quality which will not affect definition. The plates are mounted in light metal circular rims with an aperture up to $4 \frac{1}{2}$ inches. Similar screens with Bakelite rims are also supplied in Great Britain by Polarizers Ltd. Type II is intended for use over light sources and consists of a Polaroid sheet cemented to a single plate of glass. In this form the polarizer slightly diffuses the incident light, but may be had in sheets up to 12 inches square. The amount of polarization by single plates of Polaroid and the transmission of ordinary light through two films parallel and crossed as well as two plates parallel and crossed has been determined by L. R. Ingersoll, J. G. Winans and E. H. Krause ${ }^{5}$ for wave-lengths $4000 \mathrm{~A}$. to $20,000 \mathrm{~A}$. and by J. Strong ${ }^{5}$ for wavelengths $3000 \mathrm{~A}$. to $11,000 \mathrm{~A}$.

As the behaviour of this new material cannot be judged without some knowledge of such measurements, the following table gives the average figures taken from the curves obtained by these authors. There appears to be some variance with different samples.

The dichroic properties of the material can be plainly seen by the smaller percentage of polarized light in the violet and red ends of the spectrum as compared with the intermediate portions where the absorption of one ray is almost complete. Consequently when a bright light is observed 
through two crossed plates, it is seen to be coloured a deep red.

The transparency of films and plates to ordinary light is also not without interest. The material is opaque to the ultra-violet but very transparent to the infra-red, when parallel or crossed, so that a pair of crossed films will serve as an excellent infra-red filter and will not polarize the transmitted radiation. The transparency of films in the visible spectrum is not so great as that of plates, due apparently to greater scatter, but of course the glass in the plates absorbs a large percentage of the infra-red.

\begin{tabular}{|c|c|c|c|c|c|}
\hline & \multirow{2}{*}{$\begin{array}{l}\text { Percentage } \\
\text { Polarization }\end{array}$} & \multicolumn{4}{|c|}{ Percentage Transmission } \\
\hline & & $\begin{array}{r}\text { Fi } \\
\text { Parallel }\end{array}$ & Crossed & $\begin{aligned} \text { Pla } \\
\text { Parallel }\end{aligned}$ & Crossed \\
\hline $\begin{array}{l}3000 \mathrm{~A} . \\
4000 \\
4500 \\
5000 \\
5500 \\
6000 \\
6500 \\
7000 \\
8000 \\
9000 \\
1 \mu \\
1 \cdot 1 \mu \\
1 \cdot 5 \mu \\
2 \cdot 0 \mu\end{array}$ & $\begin{array}{l}\overline{70} \\
70 \\
95 \\
98 \\
98 \\
96 \\
91 \\
32 \\
9 \\
5 \\
1 \\
0.5\end{array}$ & $\begin{array}{l}0 \\
1 \cdot 5 \\
12 \\
13 \\
15 \\
16 \\
25 \\
34 \\
63 \\
77 \\
85 \\
85 \\
-\end{array}$ & $\begin{array}{l}0 \\
0 \\
0 \cdot 5 \\
0 \\
0 \\
0 \\
0 \\
2: 5 \\
62 \\
77 \\
85 \\
85 \\
= \\
-\end{array}$ & $\begin{array}{r}0 \\
22 \\
28 \\
32 \\
33 \\
33 \\
33 \\
32 \\
28 \\
37 \\
39 \\
41 \\
-\end{array}$ & $\begin{array}{l}0 \\
5 \\
4 \\
3 \\
2 \cdot 5 \\
2 \cdot 5 \\
2 \cdot 5 \\
2 \cdot 5 \\
5 \\
5 \\
29 \\
43 \\
41 \\
-\end{array}$ \\
\hline
\end{tabular}

In a subsequent patent specification ${ }^{6}$, Land describes the use and preparation of films of nitrocellulose containing polarizing ultra-microscopic particles of inorganic periodides, particularly purpureocobaltchloridesulphateperiodide. He states that films prepared with particles of this substance, suitably oriented, give complete polarization, with a colourless transmitted beam and extremely small loss by absorption. The material should be a great improvement on the Herapathite material.

The scientific and technical applications of Polaroid are almost unlimited. The most obvious and most important application which first springs to the mind and which is specifically mentioned by Land in his first patent specification is to the vexed problem of glare from the headlights of motor vehicles. By covering the aperture of the head lamps with Polaroid with its plane of polarization in a specified position, parallel say to the vertical plane, then an observer looking through a Polaroid screen with its plane of polarization parallel to the horizontal plane will see the head lamp merely as a faint dark red source, but all objects illuminated by the headlight will be seen almost as clearly as without the screen. The light from the head lamp is polarized with its intensity reduced by a little more than fifty per cent, but this polarized light is depolarized into ordinary light when diffusely reflected from the surfaces of objects, the visibility of which is therefore unimpaired by the observer's crossed screen.

To be effective as an anti-glare device on the road, it would be necessary to enforce the use and the manner of using this material by legislation. But it is not manufactured in Great Britain, and, moreover, the patent rights are in the hands of one body which is very much alive to the immense value of this epochal invention.

Since ordinary light when specularly reflected from non-metallic surfaces at about $32^{\circ}$ to $37^{\circ}$ to the surface is strongly polarized in the plane of incidence, that is, the vibrations are parallel to the surface, it will be absorbed by transmission through a Polaroid film with its plane of polarization parallel to the surface. Consequently the details of reflecting objects can be seen more clearly through Polaroid films and are invaluable in photographic work. A Pola screen placed in front of the lens of a camera will enable otherwise impossible photographs to be taken obliquely through glass or water. Oblique reflections which hide surface detail or interfere with good composition can be subdued.

A single screen used in the form of spectacles will give the observer all the above advantages and aid his view of glazed pictures obstructed by surface reflections in picture galleries. But such spectacles will not cut out effectively the surface glare of roads from the setting sun which is so troublesome when motoring, since such oblique reflections are scarcely polarized.

If, however, a second screen adjustable by rotation is used in the spectacles a variable antiglare goggle is at once available, and has been described by $\mathbf{E}$. H. Land in a patent specification? The screens arranged in this way can be used as a practically neutral variable density device of great simplicity and utility in many applications. The light scattered from the blue sky in and about a plane perpendicular to the sun's rays is strongly polarized in the plane containing the sun and the ray from the sky. Consequently the tone of a clear blue sky in a direction perpendicular to the sun's rays can be varied in photography from light to very dark by rotation of a Pola-screen placed in front of the lens, and surrounding objects can be made to stand out in a remarkable manner.

When the illumination is plane polarized by placing a Pola screen type II over the light source, various useful effects can be obtained by observation or photography through a second screen. Though polarized light becomes ordinary light when diffusely reflected, it retains its polarization when specularly reflected from non-metallic surfaces, and troublesome reflections can be cut out by the second screen. 
Reflection from metallic surfaces, however, is more complicated. Common light is only partially polarized by metallic reflection, and plane polarized light is reflected as such only when the plane of polarization is in or perpendicular to the plane of incidence. At all other azimuths, plane polarized light is converted into elliptically polarized light by metallic reflection and so the second screen cannot completely cut out such reflections.

The advantage of polarized illumination depends upon the fact that specular reflections can be quenched by the second screen but reflections depolarized by scatter pass through it. When semi-transparent objects are examined or photographed in polarized light, the surface specular reflections can be quenched, but the light diffused from deeper layers passes the second screen and such objects assume a new appearance. Thus the true texture of the skin becomes more apparent, when the illumination is intense.

It has been stated that stereoscopic cinema projection was demonstrated by the inventor of Polaroid in New York a short time ago. For this purpose the two stereoscopic film pictures are projected on to the same screen one over the other, through Polaroid plates with their planes of polarization set at right angles to one another. The audience were supplied with Polaroid spectacles with the plate over the left eye set to cut out the right-eye picture and that over the right eye set to cut out the left-eye picture. But the nature of the projection screen surface has not been disclosed. The usual interference colour effects produced by introducing plates of uniaxial crystals or substances such as Cellophane which behave optically like uniaxial crystals, between two crossed Polaroid screens, might be used for the projection of coloured backgrounds of changing hue on the stage.

The Polaroid screen will also find many scientific uses, more particularly if the somewhat fanciful prices at present demanded are very considerably reduced when mass production starts. It cannot replace the nicol in measuring instruments in which the polarization of the light must be complete, but obviously it can be used with great advantage in photo-elastic apparatus, strain viewers, and projecting lanterns, microscopes, telescopes, ophthalmic instruments in which the nicol restricts the aperture or interferes with the definition of the optical image.

Now that E. H. Land has so successfully demonstrated the possibility of manufacturing polarizing screens, without doubt attention will be concentrated upon the production of more transparent material, transmitting a higher percentage of plane polarized light uniformly throughout the visible spectrum. It would seem that when the remarkable phenomena of photoanisotropy observed by Weigert and by Zocher and Coper in recent years are more fully understood, means will be found by which we may completely control the production of plane, circularly or even elliptically polarized light.

Weigert discovered that plane polarized light may turn an isotropic solid colloidal system into an anisotropic one with double refracting and dichroic properties. By exposing a dry layer of silver chloride suspended in gelatine to common light a bluish-red photochloride is formed. If now the photochloride is exposed to intense polarized red light the photochloride becomes double refracting and dichroic, with the plane of polarization parallel to that of the exciting light. Violet or ultra-violet light has no such effect, neither does the photoanisotropy develop at very low temperatures. Werner and Kuhn have found also that photoanisotropy is produced in an aqueous gel of the dyestuff cotton yellow when excited by plane polarized light.

But Zocher and Coper have found that circularly polarized light will convert thin photochloride layers prepared by chlorinating silver mirrors into layers exhibiting circular dichroism and circular double refraction. The circular dichroism produced is of the same sign as the dichroism produced by plane polarization. That is to say, the photoanisotropic layer is more transparent to circularly polarized light of the same sense as the exciting red light than to circularly polarized light of the opposite sense.

Circular double refraction in which the circularly polarized wave of one sense is retarded relatively to the wave of the opposite sense of rotation is simply optical activity, and this is the first case in which optical activity has been produced by light itself.

For those who are not fully acquainted with these interesting and important investigations on photoanisotropy it might be mentioned that a brief and lucid description of them has been given by Prof. H. Freundlich ${ }^{8}$, with an extensive bibliography.

\footnotetext{
${ }^{1}$ Phil. Mag., 3, 161 (1852) ; 6, 346 (1853); 7, 352 (1854) ; 9, 366 (1855).

" "How to Work with the Microscope", by Lionel S. Beale. 5th edn., 1880 , p. 23 .

3 "The Microscope and its Revelations", by William B. Carpenter. 2nd edn., 1857, pp. 127, 128.

- British Patent No. 412,179. Dec. 16, 1932.

${ }^{5}$ J. Opt. Soc. Amer., 26, p. 233 and p. 256 (1936).

- British Patent No. 433,455. Jan. 15, 1934.

7 British Patent No. 442,825. June 17, 1935.

${ }^{8}$ Photographic J., 76, 395 (1936).
} 